\title{
On the form of psychometric functions for taste*
}

\author{
DENNIS McFADDEN and ROBERT B. SKINNER, JR. $\dagger$ \\ University of Texas, Austin, Texas 78712
}

\begin{abstract}
Psychometric functions were determined for the discrimination of weak solutions of quinine hydrochloride or of hydrochloric acid from distilled water. The slopes of these functions were compared with those of some functions previously determined for sodium chloride and for sucrose. In general, the functions for $\mathrm{QHCl}$ were the least steep and those for $\mathrm{NaCl}$ were the most steep, but the differences were not great. Thus, it appears that the psychometric functions for these different taste qualities have substantially the same form, even though absolute sensitivity varies over several orders of magnitude.
\end{abstract}

As part of a previous experiment (McFadden, Barr, \& Young, 1971), we obtained psychometric functions for the discrimination of weak solutions of $\mathrm{NaCl}$ or of sucrose from distilled water. The data were obtained with a forced-choice technique, and they were plotted as percentage of correct decisions against concentration in millimoles, scaled linearly. When so plotted, the functions for $\mathrm{NaCl}$ were about 10 times more steep than those for sucrose, and we pointed with interest to this remarkable difference in slope. However, we have since had second thoughts about the coordinate system used for plotting these data.

For vision and audition, it is conventional to plot psychometric data using a logarithmic measure of stimulus strength on the abscissa. This convention stems in part from the knowledge that these two systems incorporate logarithmic-like transformations at one or more junctures. That is, a logarithmic representation is judged to be a more appropriate characterization of the stimulus than is a linear one because it is more like the effective stimulus being operated on by the nervous system.

For taste, it is unusual to see psychometric data (indeed, it is just that vacuum that we have been attempting to fill), so a set of traditions similar to those that exist for other modalities does not exist for taste psychometric data. In our previous paper, the use of a linearly scaled abscissa did not stem so much from a conscious decision about the appropriateness of this choice, as from the apparent "naturalness" of a linear scale when working with a linear measure of stimulus magnitude, such as strength of solution in millimoles. However, it is clear, just from the psychophysical measurements made on suprathreshold taste stimuli, that logarithmic-like transformations occur for this modality also (see, for example, Meiselman, 1971; Moskowitz, 1970; Stevens, 1969). Thus, it is possible to argue that for taste, just as for vision and audition, logarithmic

*This research was supported in part by USPHS Grant NS 08754 from the National Institute of Neurological Diseases and Stroke. We are grateful to $K$. Gummerman for his comments and suggestions about data presentation, and for his criticisms of a preliminary draft.

Present address: University of Texas Medical Branch at Galveston. measures of stimulus strength are more appropriate than are linear measures. Of course, in the absence of a logarithmic unit of measure, the same effect can be achieved by using a linear measure scaled logarithmically. Had we done this with our previous data, the $\mathrm{NaCl}$ functions would have been only slightly more steep than the sucrose functions, an outcome more in line with data from other modalities. Here we again show the $\mathrm{NaCl}$ and sucrose data, now plotted logarithmically, and we also report new data taken with two other taste substances-hydrochloric acid $(\mathrm{HCl})$ and quinine hydrochloride (here designated $\mathrm{QHCl}$ ). Thus, we now have psychometric data for all four taste qualities-salty, sweet, sour, and bitter-and we now feel that we have a better way of characterizing stimulus magnitude for these taste substances. The result is that it is now possible to be somewhat more specific about differences in the slopes of the psychometric functions for various taste stimuli.

\section{METHOD}

The experimental procedure was essentially the same as for the previous experiment. The data for both $\mathrm{HCl}$ and $\mathrm{QHCl}$ were collected with a yes-no forced-choice procedure. Thus, no "thresholds" were determined as such, but rather, the ability to discriminate between distilled water and a weak solution of $\mathrm{HCl}$ or of QHCl was measured for each of several strengths of the solutions.

The Ss were young undergraduates who were paid an hourly wage. Regrettably, all of the Ss were not able to serve in both the $\mathrm{QHCl}$ and the $\mathrm{HCl}$ segments of the experiment.

The $S$ and $E$ were seated on opposite sides of a wooden partition, which had a small slot along the bottom edge. The $S$ could not see the $E$ across or under the partition, and curtains prevented his looking around the anechoic chamber that housed the experiment. Every $20 \mathrm{sec}$, the E passed a 2-oz piastic cup through the slot to the $S$. The cup contained a $10-\mathrm{ml}$ sample of either distilled water (no-signal trial) or distilled water plus a small quantity of solute (signal trial). The $\mathrm{S}$ sipped the sample. held it in his mouth for approximately $5 \mathrm{sec}$, and then spit it into a large bottle at his side. He then responded, "Yes, that was a signal trial," or, "No, that was distilled water," by passing one of two small cards through the stot toward the $\mathrm{F}$. The $\mathrm{I}$ : recorded the response and then passed through the slot a similur card, which indicated whether the trial had in fact been a signal or a no-signal trial. That is, the $S$ received trial-by-trial feedback about the correctness of his responses. Response and feedback 

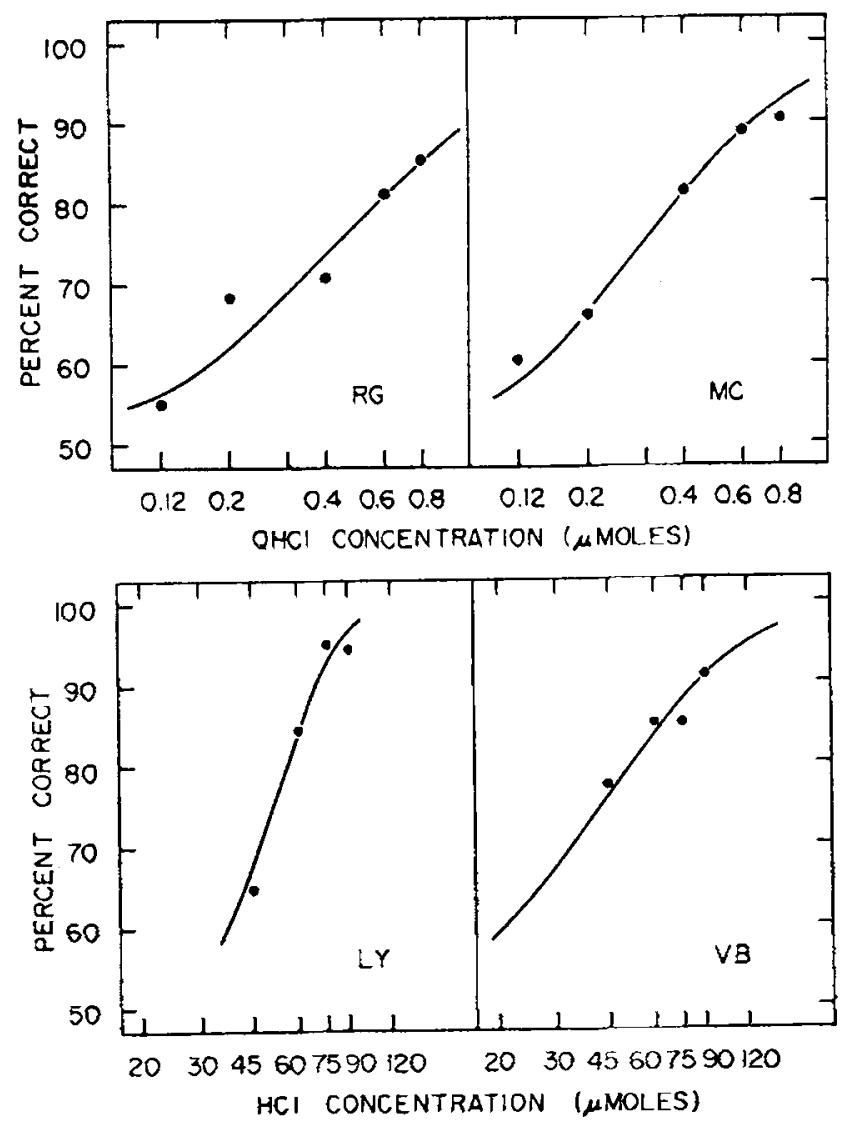

Fig. 1. Psychometric functions for detection of $\mathrm{QHCl}$ (top) and of $\mathrm{HCl}$ (bottom). The data were obtained with a yes-no method. Each point is a median based upon 12 20-trial blocks. The ogives were derived from the normal curve, and they were fitted by eys

took approximately $10 \mathrm{sec}$; at the end of this time, the $S$ thoroughly rinsed his mouth for about $5 \mathrm{sec}$ with approximately $20 \mathrm{ml}$ of distilled water, spit it into a large bottle, and then immediately took the next cup from the E. A clock with a sweep second-hand was used by the $S$ as an aid in keeping to this schedule of $20 \mathrm{sec}$ per trial.

A block of trials consisted of 20 trials, and a daily session consisted of four (for $\mathrm{HCl}$ ) or five (for QHCl) blocks, one at each of the different molarities. The order of presentation of the different molarities was varied from session to session in a pseudorandom order. Between blocks, there were rest periods lasting 2-4 min. Approximately 5-10 $\mathrm{min}$ before a block of trials was run, a second $E$ prepared a tray of 20 cups according to the master protocol; in this way, both the $S$ and $E$ were kept ignorant of the molarity being tested on any given block. The sequence of trials within each block was made up from a table of random numbers, the only constraints being that there could not be more than five consecutive trials of one type. The a priori probability of a signal trial was 0.5 , and the Ss were informed of this fact. Before the first block of the session, the $S$ was given four consecutive signal trials at the molarity scheduled for the first block. Prior to all subsequent blocks of the session, only two signal trials were given, at the appropriate molarity. The Ss were instructed not to eat or drink for at least $1 \mathrm{~h}$ prior to the daily session, and an attempt was made to run each $S$ at approximately the same time every day. None of the Ss were smokers.

The water used in this experiment, both for mixing samples and for rinsing, was kept at room temperature. Temperature readings of individual samples were taken throughout the experiment; these readings ranged from $20^{\circ}$ to $24^{\circ} \mathrm{C}$ across blocks, but the range was always less than $1^{\circ} \mathrm{C}$ within a block.

Prior to the beginning of both the $\mathrm{HCl}$ and the $\mathrm{QHCl}$ segments of the experiment, there were approximately 2 weeks of practice. During this time, the Ss learned about the procedure, and the $E$ established the range of molarity necessary to encompass chance and perfect performance. The Ss did not know that these were simply practice periods.

For both $\mathrm{HCl}$ and QHCl, 12 20-trial blocks were obtained for each of several levels of concentration. For each block of trials, a version of proportion correct was computed $\left[\mathrm{P}(\mathrm{C})_{2} ; \mathrm{McFadden}\right.$, 19701 , and psychometric functions were plotted by calculating a median value of proportion correct for each $\mathrm{S}$ for each concentration level. Medians were used because the detection task is not an easy one, and even highly experienced Ss can occasionally "get lost" in a particular block, finishing with a chance or even a below-chance score.

\section{RESULTS AND DISCUSSION}

Some of the psychometric functions obtained in this experiment are shown in Fig. 1. The data in the top panel are for $\mathrm{QHCl}$ and those in the bottom panel are for $\mathrm{HCl}$. Figure 2 shows some of the $\mathrm{NaCl}$ and sucrose data from the previous experiment. Here, however, they are plotted with a logarithmically scaled abscissa, and to be
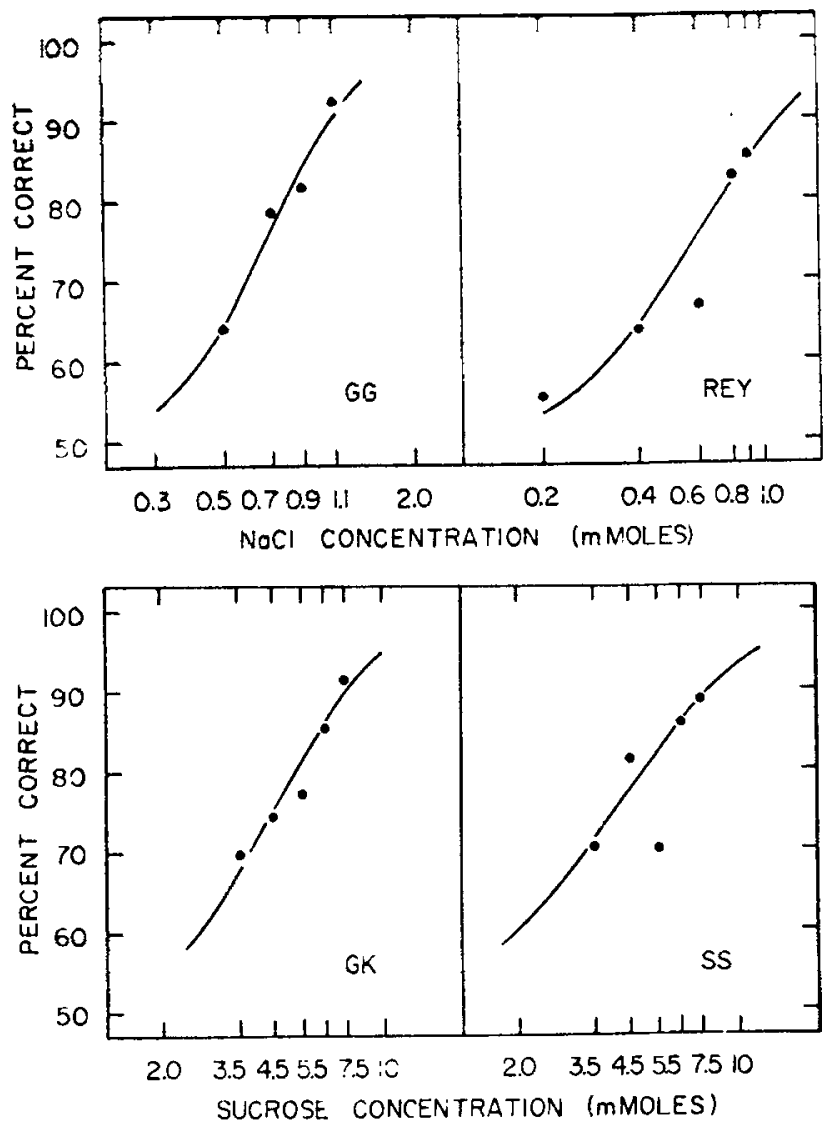

Fig. 2. Psychometric functions for detection of $\mathrm{NaCl}$ (top) and of sucrose (bottom). The data were obtained with a yes-no method. Each point is a median based upon 12 20-trial blocks. The ogives were derived from the normal curve, and they were fitted by eye. 
consistent with the data in Fig. 1, we have plotted medians rather than means. For both Figs. 1 and 2, all ordinates and abscissas are scaled exactly the same.

Table 1 summarizes all the psychometric data we have taken. Each S's data were plotted in a semilogarithmic coordinate system, and normal ogives were then fitted to the data by eye. Three values were then read from the fitted curves for each psychometric function-the stimulus concentrations necessary for $70 \%, 80 \%$, and $90 \%$ correct detections-and these values are shown in the first three columns of Table 1. As can be seen, sensitivity was greatest for $\mathrm{QHCl}$ and least for sucrose.

One approach to answering the question of how similar the slopes of the functions for the various taste qualities are is to estimate what fraction of a log unit of change in stimulus strength is required for a fixed change in performance. Such estimates appear in the last column of Table 1. They were calculated by first determining the ratio of the molarities required for $90 \%$ and $70 \%$ correct detections and by then taking the logarithm of this ratio. Of course, this is algebraically equivalent to reading off the logarithms of the two molarities and subtracting the value required for $70 \%$ correct from that for $90 \%$ correct. Either way, the result is the fraction of a log unit the solution concentration must be increased for performance to increase from $70 \%$ to $90 \%$ correct detections. As the table shows, the estimates for $\mathrm{QHCl}$ are in general larger than those for the other taste substances; this means that the psychometric functions for $\mathrm{QHCl}$ are less steep than the others. In general, the steepest psychometric functions appear to be those for $\mathrm{NaCl}$, although one of the $\mathrm{Ss}$ participating in the $\mathrm{HCl}$ segment of the experiment needed only about 0.17 of a log unit change in solution concentration to achieve the fixed change in performance. Interestingly, magnitude-estimation experiments using a "sip procedure" similar to ours also yield data that are, in general, steepest for $\mathrm{NaCl}$ and least steep for QHCl (see Meiseiman, 1971, Table 1).

It is important to note that this method for characterizing the steepness of psychometric functions ignores any absolute differences in sensitivity that may exist, and in the present case some of these differences are enormous. For example, all of the $\mathrm{Ss}$ in the $\mathrm{QHCl}$ segment of the experiment were able to achieve $80 \%$ correct detections with solution concentrations that were fractions of a micromole, whereas several millimoles were required for sinilar performance with sucrose. Obviously, were a single, linearly scaled abscissa used to portray all of these psychometric functions, some would appear as vertical lines.

If the molarity necessary for $75 \%$ or $80 \%$ correct detections is taken as a kind of "threshold," then the sensitivity of the present $\mathrm{Ss}$ to $\mathrm{QHCl}$ is greater than the typically cited values. In Pfaffman's (1959) summary table for bitter thresholds, the median threshold value for $\mathrm{QHCl}$ is $30 \mu \mathrm{M}$; the present $\mathrm{Ss}$ are all about two
Table 1

Absolute Sensitivity Data for Four Taste Substances

\begin{tabular}{llcccc}
\hline \multicolumn{5}{c}{ Molarity } & \multicolumn{2}{c}{ Required for } \\
& & $70 \%$ & $80 \%$ & $90 \%$ & Log* \\
\hline \multirow{4}{*}{ QHCl } & RG & 0.32 & 0.58 & 1.15 & 0.55 \\
& BB & 0.28 & 0.44 & 0.75 & 0.44 \\
& MC & 0.22 & 0.36 & 0.62 & 0.44 \\
& VB & 0.12 & 0.18 & 0.30 & 0.39 \\
$\mathrm{HCl}$ & VB & 35 & 53 & 87 & 0.40 \\
& LY & 48 & 57 & 70 & 0.17 \\
& GG & 0.59 & 0.78 & 1.10 & 0.27 \\
$\mathrm{NaCl}$ & REY & 0.50 & 0.73 & 1.10 & 0.34 \\
& EAB & 0.43 & 0.60 & 0.88 & 0.31 \\
& JK & 2.70 & 3.40 & 4.50 & 0.22 \\
& GK & 3.8 & 5.3 & 7.7 & 0.31 \\
\multirow{5}{*}{ Sucrose } & SS & 3.3 & 5.0 & 8.1 & 0.39 \\
& EAB & 2.9 & 4.2 & 6.5 & 0.35 \\
& PH & 5.3 & 7.8 & 12.0 & 0.35 \\
\hline
\end{tabular}

Note-For $\mathrm{QHCl}$ and $\mathrm{HCl}$ the data are given in units of micromoles and for $\mathrm{NaCl}$ and sucrose in units of millimoles. * Log (molarity at 90\%/molarity at $70 \%$ ).

orders of magnitude more sensitive than this. Of course, the difference may lie with the psychophysical method rather than with the particular Ss employed. For $\mathrm{HCl}$, the present Ss are at the lower end of the range of values cited by Pfaffman. As previously noted, our estimates of sucrose and $\mathrm{NaCl}$ sensitivity are also in good agreement with classical results.

There is precedent for comparing the slopes of psychometric functions independent of the absolute values of the stimuli necessary to obtain them. For audition, the conclusion has been that for a large number of listering conditions, the functions are of the same form and steepness (Egan, Lindner, \& McFadden, 1969; Green \& Swets, 1966, Chap. 7; but compare Watson, Franks, \& Hood, 1972). The same conclusion seems to be appropriate for these taste data as well. For, while there are some differences obvious in Table 1, they certainly are not large-particularly in light of the enomous differences that exist in absolute sensitivity for the different substances. Also, were data taken from more Ss, it is possible that the range of individual differences might prove to be larger than the differences across taste substances. For these reasons, we now believe that the best working hypothesis is that the psychometric functions for the different taste qualities are of essentially the same form and slope. Thus, we retract our previous assertion that the functions for $\mathrm{NaCl}$ are many times more steep than those for sucrose. The assertion was based on linear thinking.

\section{REFERENCES}

Egan, 3. P.. Lindner, W. A., \& McFadden, D. Masking-level differences and the form of the psychometric function. Perception \& Psychophysics, 1969, 6, 209-215.

Green. D. M. \& Swets, J. A. Signal detection theory and psychophysics. New York: Wiley, 1966.

persions of proportion correct for use in forced-choice experiments. Perception \& Psychophysics, 1970, 8, 336-342. 
McFadden, D., Barr, E. A., Y Young, R. E. Audio analgesia: Lack of a cross-masking effect on taste. Perception \& Psychophysics, 1971, 10, 175-179.

Meiselman, H, L. Effect of presentation procedure on taste intensity functions. Perception \& Psychophysics, 1971, 10 , 15-18.

Moskowitz, H. R. Ratio scales of sugar sweetness. Perception \& Psychophysics, 1970, 7, 315-320.

Pfaffman, C. The sense of taste. In J. Field (Ed.), Handbook of physiology. Vol. 1. Baltimore: Williams \& Wilkins, 1959. Chap. 20.
Stevens, S. S. Sensory scales of taste intensity. Perception \& Psychophysics, 1969, 6, 302-308.

Watson, C. S., Franks, J. R., \& Hood, D. C. Detection of tones in the absence of external masking noise. I. Effects of signal intensity and signal frequency. Jourmal of the Acoustical Society of America, 1972,52,634-643.

(Received for publication August 22, 1973; accepted December 8, 1973.) 